\title{
NON HOMOGENEOUS DIRICHLET PROBLEM FOR THE KDVB EQUATION ON A SEGMENT
}

\author{
ISAHI SÁnCHEZ SuÁreZ, GERARdo LORETO GómEZ \\ AND MARCELA MORALES MORFÍN
}

Abstract. We study the Non homogeneous Dirichlet problem with large initial data for the KdVB equation on the interval $x \in(0,1)$

$$
\left\{\begin{array}{c}
u_{t}+u_{x} u-u_{x x}+u_{x x x}=0, t>0, x \in(0,1) \\
u(x, 0)=u_{0}(x), x \in(0,1) \\
u(0, t)=u(1, t)=0, t>0 \\
u_{x}(1, t)=h(t), t>0
\end{array}\right.
$$

We prove that if the initial data $u_{0} \in \mathbf{L}^{2}$ and boundary data $h(t) \in \mathbf{H}_{\infty}^{1}(0, \infty)$ then there exist a unique solution $u \in \mathbf{C}\left([0, \infty) ; \mathbf{L}^{2}\right) \cup \mathbf{C}\left((0, \infty) ; \mathbf{H}^{1}\right)$ of the initial-boundary value problem (1). We also obtain the large time asymptotic of solution uniformly with respect to $x \in(0,1)$ as $t \rightarrow \infty$.

Mathematics subject classification (2010): 35Q35.

Keywords and phrases: dissipative dispersive nonlinear equation, large time asymptotics, KdVB equation.

\section{REFERENCES}

[1] F. Achleitner, F.; C.M. Cuesta, S. Hittmeir, Travelling waves for a non-local Korteweg-de Vries-Burgers equation, J. Differ. Eq. 257, 3 (2014), 720-758.

[2] H. AiKawa AND N. HAYASHI, Holomorphic solutions of semilinear heat equations, Complex Variables, 16 (1991), 115-125.

[3] H. Aikawa, N. Hayashi And S. SAitoh, The Bergman space on a sector and the heat equation, Complex Variables, 15 (1990), 27-36.

[4] C. J. Amick, J. L. Bona And M. E. Schonbek, Decay of solutions of some nonlinear wave equations, J. Diff. Eq. 81 (1989), 1-49.

[5] P. BILlER, Asymptotic behavior in time of solutions to some equations generalizing the Korteweg-de Vries-Burgers equation, Bull. Polish. Acad. Sc. Math., 32 (1984), 275-282.

[6] J. L. BONA AND L. LUO, More results on the decay of the solutions to nonlinear dispersive wave equations, Discrete and Continuous Dynamical Systems, 1 (1995), 151-193.

[7] X. Deng, W. Chen, J. Zhang, Boundary control of the Korteweg-de Vries-Burgers equation and its well-posedness. Int. J. Nonlinear Sci. 14, 3 (2012), 367-374.

[8] D. B. DIX, The dissipation of nonlinear dispersive waves; the case of asymptotically weak nonlinearity, Comm. P.D.E., 17 (1992), 1665-1693.

[9] T. Dlotкo, The generalized Korteweg-de Vries-Burgers equation in $H^{2}(\mathbb{R})$, Nonlinear Anal. 74, 3 (2011), 721-732.

[10] T. DlotKo, C. Sun, Asymptotic behavior of the generalized Korteweg-de Vries-Burgers equation, J. Evol. Equ. 10, 3 (2010), 57-595.

[11] G. GAO, A theory of interaction between dissipation and dispersion of turbulence, Sci. Sinica (Ser. A) 28 (1985), 616-627. 
[12] N. HAYASHI, E. I. KAIKINA, Nonlinear theory of pseudodifferential equations on a half-line, NorthHolland Mathematics Studies, 194. Elsevier Science B.V., Amsterdam, 2004.

[13] N. Hayashi, E.I. Kaikina AND I. A. Shishmarev, Asymptotics of Solutions to the BoundaryValue Problem for the Korteweg-de Vries-Burgers equation on a Half-Line, Journal of Mathematical Analysis and Applications, 265, 2 (2002), 343-370.

[14] N. HAYASHI, E. I. KAIKINA AND R. MANZO, Local and global existence of solutions to the nonlocal Whitham equation on half-line, Nonlinear Analisys, 48 (2002), 53-75.

[15] R. S. Johnson, A nonlinear equation incorporating damping and dispersion, J. Fluid Mech. 42 (1970), 49-60. Full Text via CrossRef.

[16] E.I. Kaikina, Leonardo Guardado-Zavala, Hector F. Ruiz-Paredes and S. JuarezZIRATE, Korteweg de Vries Burgers Equation on a Segment, CUBO A Mathematical Journal, Vol. 12-01, pp. 41-58. March. 2010

[17] NAUMKIN, I. P. Klein-Gordon equation with critical nonlinearity and inhomogeneous Dirichlet boundary conditions, Differential Integral Equations 29, 1-2 (2016), 55-92.

[18] NAUmKIn, I. P. Initial-boundary value problem for the one dimensional Thirring model, J. Differ. Eq. 261, 8 (2016), 4486-4523.

[19] NAUmKIn, I. P. Sharp asymptotic behavior of solutions for cubic nonlinear Schrödinger equations with a potential, J. Math. Phys. 57, 5 (2016), 051501, 31 pp.

[20] Naumkin, I. P. Cubic nonlinear Dirac equation in a quarter plane, J. Math. Anal. Appl. 434 2, (2016), 1633-1664.

[21] L. QIAN, L. TIAn, D. Zhang, Unique continuation property and decay for the Korteweg- de VriesBurgers equation with localized damping, Int. J. Nonlinear Sci. 18, 2 (2014), 110-115.

[22] M. SADIKHOV, On existence in large for almost everywhere solution of one-dimensional mixed problem for a class of Korteweg-de Vries-Burgers type nonlinear equations, Proc. Inst. Math. Mech. Natl. Acad. Sci. Azerb. 29 (2008), 157-166.

[23] I. A. Shishmarev, M. Tsutsumi And E. I. Kaikina, Asymptotics in time for the nonlinear nonlocal Schrödinger equations with a source, J. Math. Soc. Japan, 51 (1999), 463-484.

[24] P. K. Shukla And S. G. Tagare, Phys. Lett. A 59,38 (1976).

[25] L. Van Wijngandien, On the motion of gas bubbles in a perfect fluid, Ann. Rev. Fluid Mech. 4 (1972), 369-373.

[26] H. Zhang, L.J. Han, Global well-posedness and inviscid limit for the modified Korteweg-de VriesBurgers equation, Nonlinear Anal. 71, 12 (2009), e1708-e1715. 